Prevalence of HCQ Retinopathy Stratified by Cumulative Dose (g)

$\approx$ No HCQ retinopathy $\%$ HCQ retinopathy $120.0 \%$

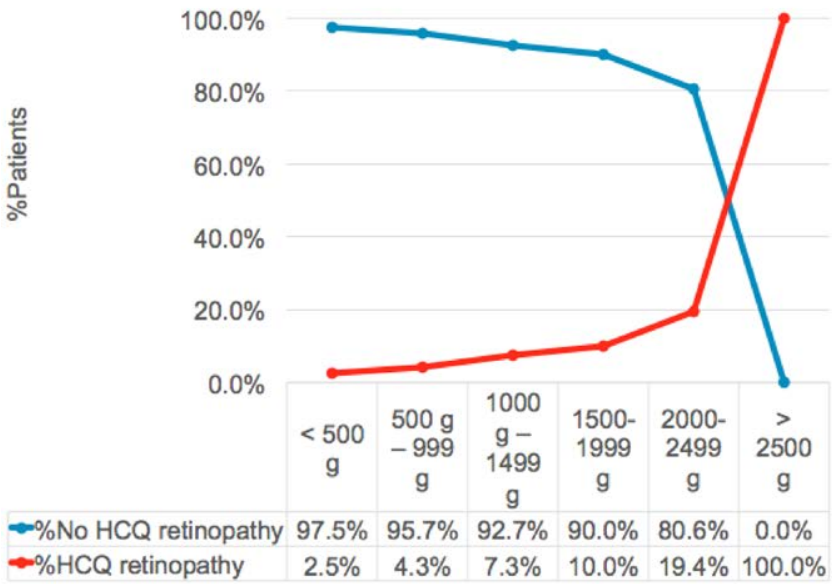

REFERENCES:

[1] Hobbs HE. Sorsby A, \& Freedman A. Retinopathy Following Chloroquine Therapy. The Lancet. 1959; 2(7101): 478-480.

[2] Levy, G. D., Munz, S. J., Paschal, J., Cohen, H. B., Pince, K. J., \& Peterson, T. Incidence of hydroxychloroquine retinopathy in 1,207 patients in a large multicenter outpatient practice. Arthritis \& Rheumatism: 1997; 40(8): 1482-1486.

[3] Ding, H. J., Denniston, A. K., Rao, V. K., \& Gordon, C. Hydroxychloroquine-related retinal toxicity. Rheumatology. 2016; 55(6): 957-967.

[4] Stelton, C. R., Connors, D. B., Walia, S. S., \& Walia, H. S. Hydrochloroquine retinopathy: characteristic presentation with review of screening. Clinical rheumatology. 2013; 32(6): 895-898.

[5] Marmor, M. F., Kellner, U., Lai, T.Y., Melles, R. B., \& Mieler, W. F. Recommendations on screening for chloroquine and hydroxychloroquine retinopathy (2016 revision). Ophthalmology. 2016; 123(6): 1386-1394

[6] Melles, R. B., \& Marmor, M. F. The risk of toxic retinopathy in patients on long-term hydroxychloroquine therapy. JAMA ophthalmology. 2014; 132(12): 1453-1460.

Disclosure of Interests: None declared

DOI: 10.1136/annrheumdis-2021-eular.328

\section{OP0134 NOVEL INTERFERON GENE EXPRESSION SCORES PREDICT REFRACTORY SEVERE CUTANEOUS DISEASE FOLLOWING RITUXIMAB THERAPY IN SLE}

L. M. Carter ${ }^{1}$, A. Alase2, Z. Wigston2, A. Psarras2, A. Burska2, M. Y. MD Yusof2, E. Hensor2, J. Reynolds ${ }^{3}$, M. Wittmann2, I. N. Bruce ${ }^{4}$, E. Vital2on behalf of The MASTERPLANS Consortium. ${ }^{1}$ University of Leeds, Leeds Institute of Rheumatic and Musculoskeletal Medicine, Leeds, United Kingdom; ${ }^{1}$ University of Leeds, Leeds Institute of Rheumatic and Musculoskeletal Medicine, Leeds, United Kingdom; ${ }^{3}$ University of Birmingham, Institute of Inflammation and Ageing, Birmingham, United Kingdom; ${ }^{4}$ University of Manchester, Division of Musculoskeletal \& Dermatological Sciences, Manchester, United Kingdom

Background: We developed and validated two continuous interferon-stimulated gene (ISG) expression scores (IFN-Score-A and IFN-Score-B) that predict clinical outcomes in SLE. IFN-Score-A includes ISGs typically present in a global interferon signature while IFN-Score-B includes additional ISGs potentially responsive to multiple IFN subtypes [1]

We have previously shown that these scores associate with treatment response following rituximab (RTX) therapy within the British Isles Lupus Assessment Group (BILAG) Biologics Register (BILAG-BR), a UK wide study of patients treated with RTX for active SLE following cyclophosphamide and/ or mycophenolate mofeti treatment failure. Specifically, multivariable analysis showed higher baseline IFNScore-B independently predicted BILAG response at 6 months post treatment [2] We also showed that response of cutaneous lupus to RTX can be poor even when other organs respond well, and that interferons are enriched in the skin of patients with SLE where dysregulated keratinocytes are a source of IFNK [3]. MASTERPLANS is a consortium aimed at identifying therapeutic biomarkers in SLE.

Objectives: To investigate how IFN-Score-A and -B associated with skin disease and response to RTX.
Methods: Pre-treatment whole blood samples were collected in TEMPUS tubes from subjects undergoing first RTX treatment within BILAG-BR. IFN-Scores were derived using a custom Taqman array as previously described [1]. Clinical response was defined as improvement in BILAG-2004 disease activity, with a maximum of one domain showing persistent BILAG-2004 grade B disease, and no new BILAG grade $A$ or $B$ disease flares at 6 months. The mucocutaneous domain of BILAG was then analysed separately.

Results: 147 patients were studied, of whom 90 had follow up data available. Baseline BILAG-2004 grade A/B disease activity predominantly affected the mucocutaneous domain in 74/147 (50.3\%), musculoskeletal in $61 / 147(41.5 \%)$ and renal domain $66 / 147(37.4 \%)$.

At 6 months 59/90 (65.6\%) achieved an overall treatment response. Responders showed significantly higher mean IFN-Score-B compared with non-responders $(-1.8$ vs $-2.4, p=0.04)$. Among those with active grade A/B BILAG-2004 mucocutaneous disease at baseline, $38 / 50(76 \%)$ showed improvement within this domain at 6 months. However, among overall non-responders, 7/31 (22.6\%) had new or residual BILAG-A mucocutaneous disease at 6 months post RTX indicating it to be a substantial component of overall treatment failure. In contrast persistent grade A musculoskeletal disease was seen in $9.7 \%$ of non-responders. BILAG-A mucocutaneous disease is characterised by severe manifestations including extensive rashes covering $>18 \%$ of body surface area, severe bullous lupus or panniculitis and disabling deep mucosal ulceration. Neither IFN-Score-A nor IFN-Score-B were significantly associated with the severity of mucocutaneous disease at baseline. However, individuals with persistent or new BILAG-A mucocutaneous disease at six months following RTX displayed significantly lower baseline IFN-Score-B than those with improving or residual less severe disease ( -3.0 vs $-2.1, p=0.04)$ after RTX

Conclusion: Low IFN score-B status identified an endotype of severe mucocutaneous SLE which was resistant to RTX therapy in the BILAG-BR cohort. We previously showed that high IFN-score-B independently predicts overall therapeutic response to rituximab. Further work will aim to refine IFN status as overall and organ specific biomarkers in SLE.

\section{REFERENCES:}

[1] El-Sherbiny et al., Sci. Rep. 2018; 8: 5793.

[2] Alase et al., ARD 2019;78:763-764

[3] Psarras et al., Nat Commun. 2020; 11: 6149

Acknowledgements: We would like to thank the Medical Research Council, National Institute of Health Research, UK for funding the MASTERPLANS project Disclosure of Interests: Lucy Marie Carter: None declared, Adewonuola Alase: None declared, Zoe Wigston: None declared, Antonios Psarras: None declared, Agata Burska: None declared, Md Yuzaiful Md Yusof: None declared, Elizabeth Hensor: None declared, John Reynolds: None declared, Miriam Wittmann Consultant of: Abbvie, Celgene, Janssen, L'Oreal, Novartis and Pfizer, lan N. Bruce Speakers bureau: GlaxoSmithKline, UCB Pharma, Consultant of: AstraZeneca, Eli Lilly, GlaxoSmithKline, ILTOO Pharma, Medlmmune, Merck Serono, Grant/ research support from: Genzyme Sanofi, GlaxoSmithKline, Edward Vital Consultant of: Roche, GSK and AstraZeneca, Grant/research support from: Roche, GSK and AstraZeneca

DOI: 10.1136/annrheumdis-2021-eular.1098

\section{OP0135 SAFETY AND EFFICACY OF SUBCUTANEOUS BELIMUMAB AND INTRAVENOUS RITUXIMAB COMBINATION IN PATIENTS WITH PRIMARY SJÖGREN'S SYNDROME: A PHASE 2, RANDOMISED, PLACEBO-CONTROLLED 68-WEEK STUDY}

X. Mariette ${ }^{1}$, C. Baldini ${ }^{2}$, F. Barone ${ }^{3}$, H. Bootsma ${ }^{4}$, K. Clark ${ }^{5}$, S. De Vita ${ }^{6}$ K. Lerang ${ }^{7}$, P. Mistry ${ }^{8}$, F. Morin ${ }^{9}$, R. Punwaney ${ }^{10}$, R. Seror ${ }^{1}$, P. L. Van Daele $^{11}$, A. Van Maurik ${ }^{12}$, N. Wisniacki ${ }^{13}$, D. Roth ${ }^{14}$. ${ }^{1}$ Université Paris-Saclay, Rheumatology, Paris, France; ${ }^{2}$ University of Pisa, Rheumatology Unit, Department of Clinical and Experimental Medicine, Pisa, Italy; ${ }^{3}$ University of Birmingham, College of Medical and Dental Sciences, Rheumatology, Birmingham, United Kingdom: ${ }^{4}$ University of Groningen, University Medical Center Groningen, Rheumatology and Clinical Immunology, Groningen, Netherlands; ${ }^{5}$ GlaxoSmithKline, Clinical Science, Stevenage, United Kingdom; ${ }^{6}$ Azienda Ospedaliera Universitaria di Udine, Rheumatology Clinic, Department of Medical Area, Udine, Italy: ${ }^{7}$ Oslo University Hospital, Department of Rheumatology, Oslo, Norway; ${ }^{8}$ GlaxoSmithKline, R\&D Biostatistics, Stevenage, United Kingdom; ${ }^{9}$ Centre de Recherche Musculo-Squelettique, Centre de Recherche Musculo-Squelettique, Trois Rivieres, Canada; ${ }^{10} \mathrm{GlaxoSmithKline,}$ Pharmaceutical Research and Development, Philadelphia, PA, United States of America: ${ }^{11}$ Erasmus Medical Center, Department of Internal Medicine and Department of Immunology, Rotterdam, Netherlands: ${ }^{12}$ GlaxoSmithKline, Clinical Pharmacology and Experimental Medicine, Stevenage, United Kingdom; ${ }^{13}$ GlaxoSmithKline, Discovery Medicine, Stevenage, United Kingdom ${ }^{14}$ GlaxoSmithKline, Research and Development, Collegeville, PA, United States of America 
Background: B-lymphocyte stimulator (BLyS) is increased in primary Sjögren's syndrome (pSS) and plays a role in the B-cell hyperactivity thought to contribute to pSS. Belimumab (BEL, anti-BLyS) and rituximab (RTX, anti-CD20) target B cells through distinct and potentially complementary mechanisms.

Objectives: To evaluate the safety and efficacy of subcutaneous (SC) BEL/intravenous (IV) RTX combination (BEL/RTX) in patients with pSS.

Methods: This Phase 2, double-blind study (GSK Study 201842; NCT02631538) randomised 86 adults with active pSS to 4 treatment arms stratified for baseline EULAR Sjögren's Syndrome Disease Activity Index (ESSDAI) scores 5-12 or >12: placebo (PBO; $N=13)$, BEL/RTX ( $N=24$; BEL $200 \mathrm{mg} \mathrm{SC}$ weekly to Week [Wk] 24 followed by weekly PBO SC to Wk 52 + RTX 1000 mg IV, Wk 8+10), BEL monotherapy ( $\mathrm{N}=24$; BEL $200 \mathrm{mg}$ SC weekly to Wk 52) or RTX monotherapy $(\mathrm{N}=25 ; \mathrm{RTX} 1000 \mathrm{mg}$ IV, Wk $8+10)$. Follow-up was at Wk 68 . Safety to Wk 68 was the primary endpoint (safety population; patients received $\geq 1$ dose of study treatment). Secondary/other endpoints (completer population; patients completed treatment and follow-up phase) were ESSDAI score, stimulated salivary flow, CD20+ B-cell count within salivary gland biopsies, patient-reported oral dryness, and EULAR Sjögren's Syndrome Patient Reported Index (ESSPRI) score.

Results: Baseline demographics and disease characteristics were similar among arms. Adverse events (AEs) were balanced across arms. Serious AEs were infrequent but occurred only in active treatment arms (Table). No unexpected safety issues were identified with BEL/RTX relative to BEL or RTX. Treatment phase and follow-up were completed by $60 / 86$ patients. ESSDAI reductions with BEL/ RTX were numerically greater over time than $\mathrm{PBO}$, with greatest difference at Wk 68 (Table), but were not differentiated from monotherapy. Stimulated salivary flow showed a trend favouring BEL/RTX vs PBO over later time points (Table). In contrast with PBO, BEL, and RTX, salivary gland biopsies from BEL/RTX showed almost complete B-cell depletion (Wk 24). There was no clear evidence for a positive effect of BEL/RTX on patient-reported oral dryness or ESSPRI score.

Table 1. Key safety endpoints and selected efficacy endpoints

\begin{tabular}{lcccc}
\hline AEs - safety population & $\begin{array}{c}\text { PBO } \\
\mathbf{( N = 1 3 )}\end{array}$ & $\begin{array}{c}\text { BEL/RTX } \\
\mathbf{( N = 2 4 )}\end{array}$ & $\begin{array}{c}\text { BEL } \\
(\mathbf{N}=\mathbf{2 4})\end{array}$ & $\begin{array}{c}\text { RTX } \\
(\mathbf{N}=\mathbf{2 5})\end{array}$ \\
\hline AEs, n (\%) & $13(100)$ & $24(100)$ & $23(96)$ & $24(96)$ \\
Drug-related AEs, n (\%) & $10(77)$ & $17(71)$ & $16(67)$ & $14(56)$ \\
AEs leading to discontinuation/ & $1(8)$ & $5(21)$ & $3(13)$ & $5(20)$ \\
$\quad$ withdrawal, n (\%) & & & & \\
SAEs, n (\%) & 0 & $3(13)$ & $2(8)$ & $4(16)$ \\
Number of SAEs & 0 & 4 & 2 & 7 \\
Deaths, n (\%) & 0 & $1(4)^{\star}$ & 0 & 0 \\
Infections and Infestations, n (\%) & $11(85)$ & $19(79)$ & $21(88)$ & $18(72)$ \\
Efficacy - completer & $\mathbf{P B O}$ & $\mathbf{B E L} / \mathbf{R T X}$ & $\mathbf{B E L}$ & $\mathbf{R T X}$ \\
$\quad$ population & $\mathbf{( N = 8 )}$ & $\mathbf{( N = 1 7 )}$ & $\mathbf{( N = 1 9 )}$ & $\mathbf{( N = 1 6 )}$ \\
ESSDAl change, LS mean (SE) from $\mathrm{BL}$ over time & & & \\
Wk 12 & $-2.00(1.449)$ & $-4.85(0.996)$ & $-3.87(0.949)$ & $-4.22(1.048)^{\S}$ \\
Wk 24 & $-2.87(1.324)$ & $-5.32(0.911)$ & $-3.87(0.869)$ & $-5.25(0.940)$ \\
Wk 52 & $-2.87(1.294)$ & $-5.67(0.890)$ & $-4.76(0.850)$ & $-4.32(0.919)$ \\
Wk 68 & $-1.75(1.400)$ & $-5.73(0.962)$ & $-3.87(0.918)$ & $-4.38(0.994)$ \\
Stimulated salivary flow (ml/min), mean (SD) & & & \\
BL & $0.47(0.247)$ & $0.71(0.629)$ & $0.43(0.329)$ & $0.62(0.621)$ \\
Wk 12 & $0.49(0.205)$ & $0.75(0.834)$ & $0.49(0.373)$ & $0.58(0.527)$ \\
Wk 24 & $0.55(0.305)$ & $0.78(0.790)$ & $0.45(0.411)$ & $0.72(0.890)$ \\
Wk 52 & $0.53(0.378)$ & $1.00(1.146)$ & $0.58(0.608)$ & $0.69(0.781)$ \\
Wk 68 & $0.36(0.163)$ & $0.88(0.817)$ & $0.52(0.450)$ & $0.73(0.785)^{\S}$ \\
\end{tabular}

${ }^{*}$ Aspiration $(n=1)$; not considered related to treatment; patient died of food aspiration; ${ }^{\dagger}$ System organ class with the highest percent of AEs; ${ }^{\ddagger}$ Analysis was performed using mixed model repeated measures; ${ }^{\S} n=15 . B L$, baseline; LS, Least square; SAEs, serious AEs; SD, standard deviation; SE, standard error

Conclusion: No unexpected safety issues were identified with BEL/RTX relative to BEL or RTX. BEL/RTX showed a trend towards improvement in ESSDAI and stimulated salivary flow over time, which was sustained post treatment. BEL/RTX depleted B cells in minor salivary gland biopsies.

Funding: GSK

Acknowledgements: Medical writing assistance was provided by Katalin Bartus, PhD, Fishawack Indicia Ltd., UK, part of Fishawack Health, and was funded by GSK.

Disclosure of Interests: Xavier Mariette Consultant of: BMS, Galapagos, Gilead, GSK, Janssen, Novartis, Pfizer, Servier, UCB, Grant/research support from: Servier, Chiara Baldini: None declared, Francesca Barone Consultant of: GSK, UCB, Roche, Actelion, Grant/research support from: GSK, UCB, Roche, Actelion, Employee of: Kintai therapeutics, Candel Therapeutics, Hendrika Bootsma Speakers bureau: BMS, Novartis, Consultant of: BMS, Roche, Novartis, Medlmmune, UCB, Servier, Grant/research support from: BMS, Roche, Ken Clark Shareholder of: GSK, Employee of: GSK, Salvatore De Vita Consultant of: GSK, Roche, Karoline Lerang: None declared, Prafull Mistry Shareholder of: GSK, Employee of: GSK, Frederic Morin: None declared, Rajesh Punwaney Shareholder of: GSK, Employee of: GSK, Raphaèle Seror Consultant of: GSK, BMS, Fresenius Kabi, Boehringer, Jansen, Amgen, Pfizer, Roche, Paul LA van
Daele: None declared, Andre van Maurik Shareholder of: GSK, Employee of: GSK, Nicolas Wisniacki Shareholder of: GSK, Employee of: GSK, David Roth Shareholder of: GSK, Employee of: GSK

DOI: 10.1136/annrheumdis-2021-eular.2170

\section{OP0136 RITUXIMAB PREVENTS THE PROGRESSION OF B-CELL DRIVEN INFLAMMATORY INFILTRATE IN THE MINOR SALIVARY GLANDS OF PRIMARY SJOGREN'S SYNDROME BY DOWNREGULATING IMMUNOLOGICAL PATHWAYS KEY IN ECTOPIC GERMINAL CENTRE ORGANIZATION: RESULTS FROM THE TRACTISS TRIAL}

E. Pontarini ${ }^{1}$, F. Chowdhury ${ }^{1,2}$, E. Sciacca ${ }^{1}$, S. Grigoriadou ${ }^{1}$, F. Rivellese ${ }^{1}$, D. Lucchesi ${ }^{1}$, K. Goldmann ${ }^{1}$, L. Fossati-Jimack ${ }^{1}$, P. Emery ${ }^{3}$, W. F. Ng ${ }^{4}$, N. Sutcliffe ${ }^{5}$, C. Everett ${ }^{6}$, C. Fernandez ${ }^{6}$, A. Tappuni ${ }^{2,5}$, M. Lewis $^{1}$, C. Pitzalis ${ }^{1}$, S. J. Bowman ${ }^{7}$, M. Bombardieri ${ }^{1}{ }^{1}$ William Harvey Research Institute, Queen Mary University of London, Experimental Medicine and Rheumatology, London, United Kingdom; ${ }^{2}$ Institute of Dentistry, Queen Mary University of London, Oral Immunobiology and Regenerative Medicine, London, United Kingdom; ${ }^{3}$ Leeds Institute of Rheumatic and Musculoskeletal Medicine, University of Leeds, Chapel Allerton Hospital, Leeds, United Kingdom; ${ }^{4}$ Translational and Clinical Research Institute, Newcastle University, Newcastle-upon-Tyne, Newcastle, United Kingdom; ${ }^{5}$ Royal London Hospital, Bart's Health NHS Trust, Rheumatology department, London, United Kingdom; ${ }^{6}$ Leeds Institute for Clinical Trials Research, University of Leeds, Clinical Trials Research Unit, Leeds, United Kingdom; ${ }^{7}$ University Hospitals Birmingham NHS Foundation Trust, Rheumatology Department, Birmingham, United Kingdom

Background: The pathogenic role of B-cells in primary Sjögren's Syndrome (pSS) is well established and B cell abnormalities. Because of the substantial role of B-cells, rituximab (RTX), a chimeric anti-CD20 monoclonal antibody, has been considered as a potential biologic disease modifying drug to reduce disease activity in pSS. To date, the TRial for Anti-B-Cell Therapy In patients with pSS (TRACTISS) is the largest multi-centre, placebo-controlled trial with RTX. Despite the unmet primary endpoints (30\% reduction in fatigue or oral dryness, measured by visual analogue scale), RTX treated patients showed an improvement in unstimulated whole salivary flow (Bowman et al. Arthritis Rheumatol 2017;69:1440-1450)

Objectives: To provide the first longitudinal transcriptomic and histological analysis at 3 time points over 48 weeks of labial SGs of pSS patients treated with RTX in comparison to placebo, from the TRACTISS cohort.

Methods: 26 pSS patients randomised to RTX or placebo arm consented for labial SG biopsies at baseline, weeks 16 and 48. Patients received two 1000mg cycles of RTX or placebo at baseline and week 24. SG focus score, inflammatory aggregate area fraction, B-cells (CD20+), T-cells (CD3+), follicular dendritic cells (FDCs) (CD21+) and plasma cells (CD138+) density were assessed by H\&E and immunofluorescence staining. The histological analysis was performed by digital imaging using QuPath software. RNA was extracted from matched labial SG lobules and sequenced with Illumina platform. A Principal Component Analysis (PCA) and features driving the PCA were investigated along with the most influential gene loadings. The limma-voom $\mathrm{R}$ pipeline was used to extract Differential Expressed Genes (DEGs) between placebo and RTX group at week 48, and gene ontology (GO) enrichment analysis performed through EnrichR to derive GO terms and pathways associated with DEGs.

Results: Placebo-treated labial SGs showed a worsening of inflammation highlighted by the increment of B-cell density, development of new FDC networks, and a higher ectopic GC prevalence at week 48, compared to RTX-treated patients. No difference in total T-cells and plasma cell infiltration was observed. RTX downregulated genes involved in immune cell recruitment and inflammatory aggregate organisation (e.g. CCR7, CCL19, CD52, and PDCD1) and gene signature-based analysis of 64 immune cell types highlighted how RTX preferentially blocked class-switched- and memory-B-cells infiltration in SGs at week 48. Pathway analyse confirmed the downregulation of leukocyte migration, $\mathrm{MHC}$ class II antigen presentation, and T-cell co-stimulation immunological pathways, such as the CD40 receptor complex pathway. The analysis of placebo SGs transcriptomic at week 48 showed a higher expression of genes linked to ectopic GC organisation, such as CXCL13, CCL19, LT $\beta$, in female compared to male subjects. Gender was confirmed as a key co-variate responsible for most of the variation in the PCA, together with the SG focus score and the foci area fraction.

Conclusion: Treatment with RTX showed beneficial effects on labial SG inflammatory infiltration in pSS, by downregulating genes involved in immune cell recruitment, activation and organisation in ectopic GCs. Class-switched-B-cells, memory-B-cells and FDC network development were primarily affected appearing to be responsible for the lack of progression in SG B cell infiltration in the RTX compared to the placebo arm in which clear worsening of SG immunopathology over 48 weeks was detected in female patients. Although a clear association with the clinical improvement in unstimulated salivary flow observed at week 48 in 